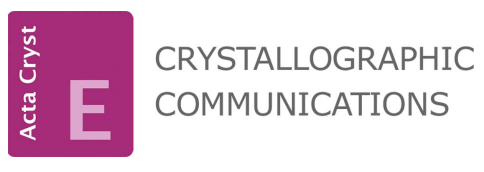

ISSN 2056-9890

Received 24 December 2018

Accepted 10 January 2019

Edited by M. Weil, Vienna University of

Technology, Austria

Keywords: crystal structure; $\mathrm{Ca}_{3} \mathrm{CoAl}_{4} \mathrm{O}_{10}$; redetermination; symmetry analysis.

CCDC reference: 1890066

Supporting information: this article has supporting information at journals.iucr.org/e

\section{Re-investigation and correct symmetry of $\mathrm{Ca}_{3} \mathrm{CoAl}_{4} \mathrm{O}_{10}$}

\author{
Volker Kahlenberg*
}

University of Innsbruck, Institute of Mineralogy \& Petrography, Innrain 52, A-6020 Innsbruck, Austria. *Correspondence e-mail: volker.kahlenberg@uibk.ac.at

A re-investigation of the crystal structure of tricalcium cobalt(II) tetraaluminate, $\mathrm{Ca}_{3} \mathrm{CoAl}_{4} \mathrm{O}_{10}$, using single-crystal $\mathrm{X}$-ray diffraction data, revealed orthorhombic $(\mathrm{Pbcm})$ symmetry. The present contribution corrects the results of a previous X-ray powder diffraction study [Vazquez et al. (2002). J. Solid State Chem. 166, 191-196] where this phase has been described in an unnecessarily low space-group symmetry $\left(P b c 2_{1}\right)$. The compound belongs to the group of tetrahedral framework structures. The distribution of the aluminium and cobalt ions among the centres of the four different tetrahedra within the asymmetric unit has been studied in detail. Charge compensation is achieved by the incorporation of two symmetrically independent calcium ions located in voids of the tetrahedral framework. $\mathrm{Ca}_{3} \mathrm{CoAl}_{4} \mathrm{O}_{10}$ is isotypic with $\mathrm{Ca}_{3} \mathrm{MgAl}_{4} \mathrm{O}_{10}$.

\section{Chemical context}

In a recent paper on the phase relationships in the system $\mathrm{CaO}-\mathrm{MgO}-\mathrm{Al}_{2} \mathrm{O}_{3}$, we reported the existence and the crystal structure of $\mathrm{Ca}_{3} \mathrm{MgAl}_{4} \mathrm{O}_{10}$ (Kahlenberg et al., 2018), a phase of interest for slags occurring in secondary refining processes in metallurgy or refractories, for example. In the course of this study it became obvious that the compound is closely related to the corresponding $\mathrm{Zn}$ and $\mathrm{Co}$ analogues that have already been reported in the literature (Barbanyagre et al., 1997; Vazquez et al., 2002). In fact Vazquez et al. (2002) used the coordinates from the $\mathrm{Zn}$ compound as a starting model for their Rietveld refinement of $\mathrm{Ca}_{3} \mathrm{CoAl}_{4} \mathrm{O}_{10}$. The major difference from our investigation on $\mathrm{Ca}_{3} \mathrm{MgAl}_{4} \mathrm{O}_{10}$ results from the fact that the previous study attributed $\mathrm{Ca}_{3} \mathrm{CoAl}_{4} \mathrm{O}_{10}$ to the acentric space group $P b c 2_{1}$, while $\mathrm{Ca}_{3} \mathrm{MgAl}_{4} \mathrm{O}_{10}$ crystallizes in the centrosymmetric space group $\mathrm{Pbcm}$. However, for the former compound the description in an acentric space group has to be scrutinized. A detailed analysis of the atomic coordinates using the program PSEUDO (Kroumova et al., 2001) indicated that the published model fulfills the symmetry requirements of Pbcm. Notably, Vazquez et al. (2002) reported problems during their structure analysis of $\mathrm{Ca}_{3} \mathrm{CoAl}_{4} \mathrm{O}_{10}$, including unstable refinements and unrealistically short cation-oxygen distances. Both observations are typical features when a structure is refined in an unnecessarily low space-group symmetry (Baur \& Tillmanns, 1986). Therefore, it was deemed appropriate to re-investigate the crystal structure of $\mathrm{Ca}_{3} \mathrm{CoAl}_{4} \mathrm{O}_{10}$ using single-crystal X-ray diffraction data obtained from melt-grown crystals. 


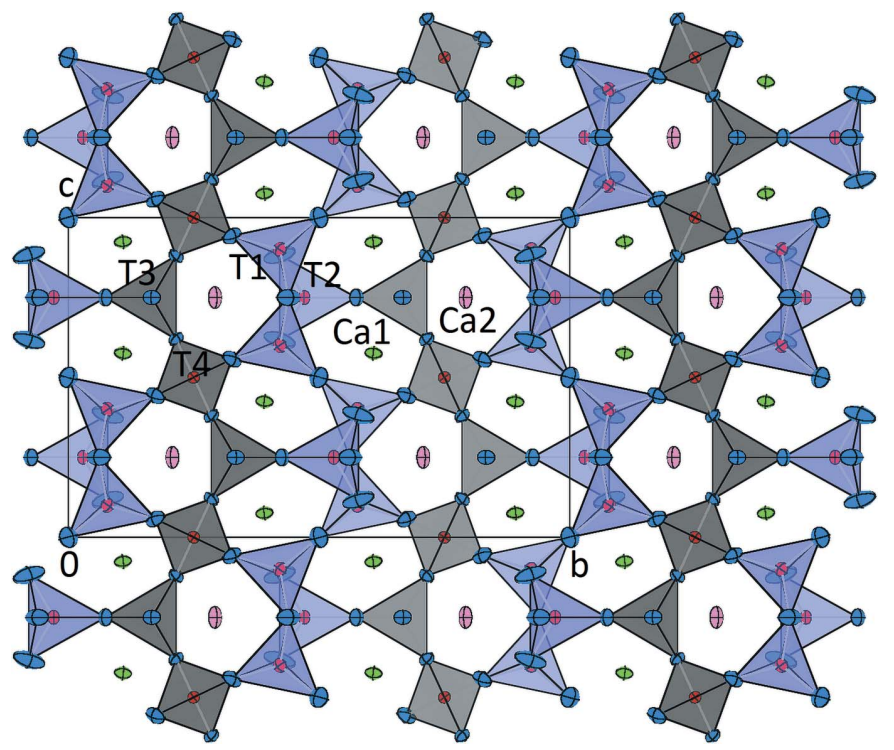

Figure 1

Projection of the crystal structure of $\mathrm{Ca}_{3} \mathrm{CoAl}_{4} \mathrm{O}_{10}$ in a view parallel to [100]. Displacement ellipsoids are shown at the $90 \%$ probability level. Colour codings for the atoms and polyhedra are as follows: blue: oxygen; red: aluminium/cobalt; $\mathrm{Ca} 1$ : pink; $\mathrm{Ca} 2$ : green; cobalt-containing tetrahedra $\left(\left[T_{10} \mathrm{O}_{4}\right]\right.$ and $\left.\left[T 2 \mathrm{O}_{4}\right]\right)$ : lilac; pure aluminium tetrahedra $\left(\left[T 3 \mathrm{O}_{4}\right]\right.$ and $\left.\left[\mathrm{THO}_{4}\right]\right)$ : grey.

\section{Structural commentary}

The crystal structure of $\mathrm{Ca}_{3} \mathrm{CoAl}_{4} \mathrm{O}_{10}$ can be described as a three-dimensional network with four symmetrically different corner-sharing $\left[(\mathrm{Al}, \mathrm{Co}) \mathrm{O}_{4}\right]$ tetrahedra around the central atoms $T 1-T 4$ (Fig. 1). The basic building units of the structure are chains of tetrahedra running parallel to [001]. Using the crystal chemical classification developed by Liebau (1985), these linear elements can be described as mixed-branched vierer single chains (Fig. 2). Condensation of adjacent chains along [010] results in the formation of stepped layers parallel to (100) (Fig. 3). Within these layers, channels can be identified which host the additional calcium ions.

Site-occupancy refinements indicated that cobalt incorporation is limited to two of the four $T$ sites within the asymmetric unit (T1 and $T 2$ ). $T 3$ and $T 4$ are virtually cobalt free. The spread of the individual $T-\mathrm{O}$ bond lengths and the $\mathrm{O}-T-\mathrm{O}$ angles follow expected crystallochemical trends. For the average $T-\mathrm{O}$ values, two groups can be distinguished: $T 1$, T2: $1.808 \AA$ and T3, T4: $1.758 \AA$. These values reflect the larger ionic radius of $\mathrm{Co}^{2+}$ for fourfold coordination $\left[r\left(\mathrm{Co}^{2+,[4]}\right): 0.58 \AA\right]$ when compared to the corresponding value for $\mathrm{Al}^{3+}\left[r\left(\mathrm{Al}^{3+,[4]}\right): 0.39 \AA\right]$ (Shannon, 1976), and can be used as an indication that $T 1$ and $T 2$ have higher Co contents. This observation compares well with the site-population refinements. Quadratic elongations as defined by Robinson et al. (1971), which can be used as numerical descriptors for the distortions, take the following values for the individual [(Al,Co) $\left.\mathrm{O}_{4}\right]$-groups: T1: 1.015, T2: 1.006, T3: 1.016, T4: 1.001.

Among the extra-framework cations, two crystallographically independent calcium sites $(\mathrm{Ca} 1, \mathrm{Ca} 2)$ can be distinguished. They are coordinated by six and eight nearest

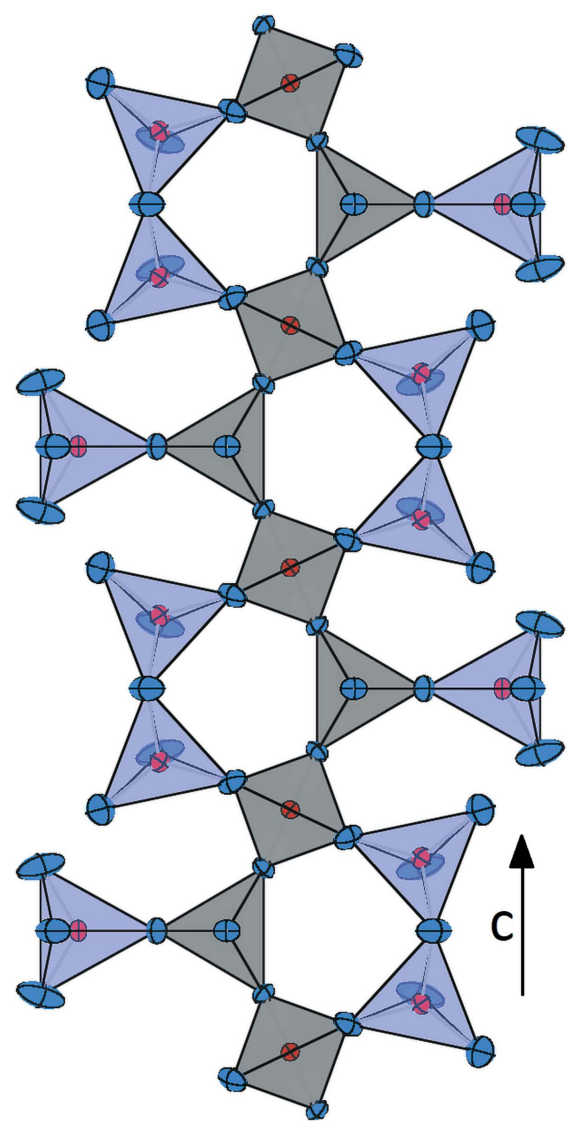

Figure 2

A single mixed-branched vierer single chain (running parallel to [001]) representing the backbone of the framework in $\mathrm{Ca}_{3} \mathrm{CoAl}_{4} \mathrm{O}_{10}$. Displacement ellipsoids as in Fig. 1.

oxygen neighbours. Their coordination polyhedra can be described as distorted octahedra and square antiprisms, respectively. Each two $\left[\mathrm{Ca}_{1} \mathrm{O}_{6}\right]$ octahedra and a single $\left[\mathrm{Ca} 2 \mathrm{O}_{8}\right]$ square antiprism form a polyhedral unit by sharing edges.

A detailed analysis of the topological features of the tetrahedral network including coordination sequences and extended point symbols has been already presented for isotypic $\mathrm{Ca}_{3} \mathrm{MgAl}_{4} \mathrm{O}_{10}$ (Kahlenberg et al., 2018) and will not be duplicated here. However, it is interesting to note that the framework consists of three (T3), four (T4) and five (T1,T2)connected tetrahedra. Notably, the net contains an $\mathrm{O}^{[3]}$-type bridging oxygen $(\mathrm{O} 3)$, simultaneously linking three tetrahedra (one $\left[\mathrm{T2O}_{4}\right]$ - and two $\left[\mathrm{THO}_{4}\right]$-units). In oxo-silicates that are

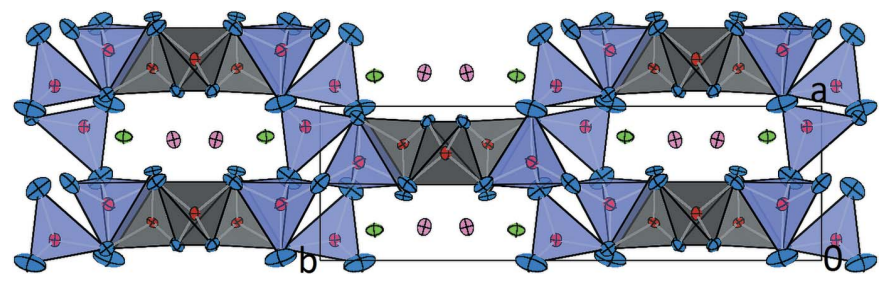

Figure 3

Projection of the crystal structure of $\mathrm{Ca}_{3} \mathrm{CoAl}_{4} \mathrm{O}_{10}$ in a view parallel to [001]. Displacement ellipsoids as in Fig. 1. 
based on $\left[\mathrm{SiO}_{4}\right]$ units, for example, only terminal $\left(\mathrm{O}^{[1]}\right)$ and simple bridging $\left(\mathrm{O}^{[2]}\right)$ oxygen atoms have been observed so far. In the present structure, the oxygen atoms $\mathrm{O} 1$ and $\mathrm{O} 2, \mathrm{O} 4$, $\mathrm{O} 5, \mathrm{O} 6, \mathrm{O} 7$ belong to these two groups. Notably, O3 is solely involved in $\mathrm{O}-T$ bonds with the two tetrahedra showing an $\mathrm{Al} /$ Co substitution.

\section{Database survey}

As mentioned above, the title compound is isotypic with $\mathrm{Ca}_{3} \mathrm{MgAl}_{4} \mathrm{O}_{10}$. For the calculation of several quantitative descriptors for the characterization of the degree of similarity between the crystal structures of $\mathrm{Ca}_{3} \mathrm{CoAl}_{4} \mathrm{O}_{10}$ and $\mathrm{Ca}_{3} \mathrm{MgAl}_{4} \mathrm{O}_{10}$, the program COMPSTRU (de la Flor et al., 2016) was employed. For the given two structures, the degree of lattice distortion S, i.e. the spontaneous strain obtained from the eigenvalues of the finite Lagrangian strain tensor calculated in a Cartesian reference system, has a value of $S=$ 0.0010. The structure of $\mathrm{Ca}_{3} \mathrm{CoAl}_{4} \mathrm{O}_{10}$ was transformed to the most similar configuration of $\mathrm{Ca}_{3} \mathrm{MgAl}_{4} \mathrm{O}_{10}$. The calculations revealed the following atomic displacements (in $\AA$ ) between the corresponding atoms in $\mathrm{Ca}_{3} \mathrm{CoAl}_{4} \mathrm{O}_{10}$ and $\mathrm{Ca}_{3} \mathrm{MgAl}_{4} \mathrm{O}_{10}$ : Ca1: 0.036; Ca2: 0.028; T1: 0.044; T2: 0.010; T3: 0.027; T4: 0.031; O1: 0.026; O2: 0.030; O3: 0.019; O4: 0.024; O5: 0.000; O6: $0.031 ; \mathrm{O} 7: 0.040$, i.e. the maximum displacement is lower than $0.05 \AA$. The measure of similarity $(\Delta)$ as defined by Bergerhoff et al. (1999) has a value of 0.007. Notably, for both structures the divalent cations $\mathrm{Co}^{2+}$ and $\mathrm{Mg}^{2+}$ are enriched in the tetrahedral positions $T 1$ and $T 2$.

The distribution of the cobalt and aluminium ions on the different $T$ sites is another difference between the new centrosymmetric model in Pbcm (this work) and the previous acentric model in $P b c 2_{1}$ (Vazquez et al., 2002). Actually, in the latter case five different tetrahedral positions have to be distinguished. The authors considered four of them to be exclusively occupied with $\mathrm{Al}$ while the remaining fifth position was attributed to be a pure cobalt site. This distribution, however, was derived from the crystal-structure refinement of the zinc analog (Barbanyagre et al., 1997) and not determined by site-occupancy refinements.

Furthermore, the new model in $\mathrm{Pbcm}$ results in considerably less distorted tetrahedra. Although soft constraints on the $\mathrm{Al}-\mathrm{O}$ and $\mathrm{Co}-\mathrm{O}$ bond lengths had been applied, individual $T-\mathrm{O}$ distances and $\mathrm{O}-T-\mathrm{O}$ angles in the $P b c 2_{1}$ structure model showed a pronounced variation between 1.68 and $2.05 \AA$ and 92.9 and $124.6^{\circ}$, respectively. The corresponding values in the present model are in the ranges from 1.719 (4) to 1.847 (2) $\AA$ and from 98.95 (16) to 120.38 (18) ${ }^{\circ}$, respectively. Finally, the displacement parameters in $\mathrm{Pbcm}$ are all well behaved, while the overall isotropic temperature factor for the oxygen atoms reported in the study of Vazquez et al. (2002) takes a physically unrealistic value of $U_{\text {iso }}=0.001$ (2) $\AA^{2}$.

\section{Synthesis and crystallization}

Single crystals of $\mathrm{Ca}_{3} \mathrm{CoAl}_{4} \mathrm{O}_{10}$ were obtained during a series of synthesis experiments in the system $\mathrm{CaO}-\mathrm{CoO}-\mathrm{Al}_{2} \mathrm{O}_{3}$.
Table 1

Experimental details.

\begin{tabular}{|c|c|}
\hline \multicolumn{2}{|l|}{ Crystal data } \\
\hline Chemical formula & $\mathrm{Al}_{4} \mathrm{Ca}_{3} \mathrm{CoO}_{10}$ \\
\hline$M_{\mathrm{r}}$ & 447.09 \\
\hline Crystal system, space group & Orthorhombic, $\mathrm{Pbcm}$ \\
\hline Temperature (K) & 293 \\
\hline$a, b, c(\AA)$ & $\begin{array}{l}5.1324(6), 16.7550(19), \\
\quad 10.6822(12)\end{array}$ \\
\hline$V\left(\AA^{3}\right)$ & $918.60(18)$ \\
\hline$Z$ & 4 \\
\hline Radiation type & Mo $K \alpha$ \\
\hline$\mu\left(\mathrm{mm}^{-1}\right)$ & 3.97 \\
\hline Crystal size $(\mathrm{mm})$ & $0.12 \times 0.09 \times 0.06$ \\
\hline \multicolumn{2}{|l|}{ Data collection } \\
\hline Diffractometer & $\begin{array}{l}\text { Rigaku Oxford Diffraction } \\
\quad \text { Xcalibur, Ruby, Gemini ultra }\end{array}$ \\
\hline Absorption correction & $\begin{array}{l}\text { Analytical (CrysAlis PRO; Rigaku } \\
\text { OD, 2015). Analytical numeric } \\
\text { absorption correction using a } \\
\text { multifaceted crystal model based } \\
\text { on expressions published by } \\
\text { Clark \& Reid (1995). }\end{array}$ \\
\hline$T_{\min }, T_{\max }$ & $0.759,0.898$ \\
\hline $\begin{array}{l}\text { No. of measured, independent and } \\
\text { observed }[I>2 \sigma(I)] \text { reflections }\end{array}$ & $5719,973,791$ \\
\hline$R_{\text {int }}$ & 0.065 \\
\hline$(\sin \theta / \lambda)_{\max }\left(\AA^{-1}\right)$ & 0.621 \\
\hline \multicolumn{2}{|l|}{ Refinement } \\
\hline$R\left[F^{2}>2 \sigma\left(F^{2}\right)\right], w R\left(F^{2}\right), S$ & $0.030,0.061,1.07$ \\
\hline No. of reflections & 973 \\
\hline No. of parameters & 96 \\
\hline No. of restraints & 1 \\
\hline$\Delta \rho_{\max }, \Delta \rho_{\min }\left(\mathrm{e} \AA^{-3}\right)$ & $0.6,-0.64$ \\
\hline
\end{tabular}

Computer programs: CrysAlis PRO (Rigaku OD, 2015), SHELXL2017/1 (Sheldrick, 2015), VESTA3 (Momma \& Izumi, 2011), publCIF (Westrip, 2010) and WinGX (Farrugia, 2012).

$1.35 \mathrm{~g}$ of the educts consisting of $\mathrm{CaCO}_{3}, \mathrm{CoO}$ and $\mathrm{Al}_{2} \mathrm{O}_{3}$ in the molar ratio 14:6:5 were homogenized in an agate mortar, transferred into a platinum crucible and covered with a lid. The container was fired in a resistance-heated furnace from 590 to $1623 \mathrm{~K}$ with a ramp of $100 \mathrm{~K} \mathrm{~h}^{-1}$. The target temperature was held for $1 \mathrm{~h}$. Subsequently, the sample was cooled down to $1273 \mathrm{~K}$ at a rate of $7.5 \mathrm{~K} \mathrm{~h}^{-1}$ and, finally, the temperature was reduced to $473 \mathrm{~K}$ at a rate of $100 \mathrm{~K} \mathrm{~h}^{-1}$. After removal of the crucible, the solidified melt cake was immediately crushed in an agate mortar and transferred to a glass slide under a polarizing binocular. A first inspection revealed the presence of two crystalline phases: larger colourless optically isotropic crystals of $\mathrm{Ca}_{3} \mathrm{Al}_{2} \mathrm{O}_{6}$ (up to $500 \mu \mathrm{m}$ in size) and considerably smaller, intensively blue birefringent crystals of $\mathrm{Ca}_{3} \mathrm{CoAl}_{4} \mathrm{O}_{10}$. A platy fragment of the latter compound showing sharp extinction under crossed polarizers was selected for further structural studies and mounted on the tip of a glass fibre using fingernail hardener as glue.

\section{Refinement}

Crystal data, data collection and structure refinement details are summarized in Table 1. Starting parameters for the atomic 
coordinates were taken from the crystal structure of $\mathrm{Ca}_{3} \mathrm{Al}_{4} \mathrm{MgO}_{10}$ (Kahlenberg et al., 2018). Initially, mixed cobalt-aluminium populations were considered for all four $T$ sites. However, the resulting values of the site occupancies for $T 3$ and $T 4$ indicated pure $\mathrm{Al}$ populations (within two standard uncertainties each). In the final cycles a restraint was introduced, fixing the total amount of cobalt distributed among the remaining $T 1$ and $T 2$ sites to four atoms per unit cell.

\section{References}

Barbanyagre, V. D., Timoshenko, T. I., Ilyinets, A. M. \& Shamshurov, V. M. (1997). Powder Diffr. 12, 22-26.

Baur, W. H. \& Tillmanns, E. (1986). Acta Cryst. B42, 95-111.

Bergerhoff, G., Berndt, M., Brandenburg, K. \& Degen, T. (1999). Acta Cryst. B55, 147-156.

Clark, R. C. \& Reid, J. S. (1995). Acta Cryst. A51, 887-897.
Farrugia, L. J. (2012). J. Appl. Cryst. 45, 849-854.

Flor, G. de la, Orobengoa, D., Tasci, E., Perez-Mato, J. M. \& Aroyo, M. I. (2016). J. Appl. Cryst. 49, 653-664.

Kahlenberg, V., Albrecht, R., Schmidmair, D., Krüger, H., Krüger, B., Tribus, M. \& Pauluhn, A. (2018). https://doi. org/10.1111/jace. 16001 Kroumova, E., Aroyo, M. I., Perez-Mato, J. M., Ivantchev, S., Igartua, J. M. \& Wondratschek, H. (2001). J. Appl. Cryst. 34, 783-784.

Liebau, F. (1985). Structural Chemistry of Silicates, p. 347. Berlin, Heidelberg, New York, Tokyo: Springer.

Momma, K. \& Izumi, F. (2011). J. Appl. Cryst. 44, 1272-1276.

Rigaku OD (2015). CrysAlis PRO. Rigaku Oxford Diffraction, Yarnton, England.

Robinson, K., Gibbs, G. V. \& Ribbe, P. H. (1971). Science, 172, $567-$ 570.

Shannon, R. D. (1976). Acta Cryst. A32, 751-767.

Sheldrick, G. M. (2015). Acta Cryst. C71, 3-8.

Vazquez, B., Torres-Martinez, L. M., Alvarez, N., Vente, J. F. \& Quintana, P. (2002). J. Solid State Chem. 166, 191-196.

Westrip, S. P. (2010). J. Appl. Cryst. 43, 920-925. 


\section{supporting information}

Acta Cryst. (2019). E75, 214-217 [https://doi.org/10.1107/S2056989019000574]

\section{Re-investigation and correct symmetry of $\mathrm{Ca}_{3} \mathrm{CoAl}_{4} \mathrm{O}_{10}$}

\section{Volker Kahlenberg}

\section{Computing details}

Data collection: CrysAlis PRO (Rigaku OD, 2015); cell refinement: CrysAlis PRO (Rigaku OD, 2015); data reduction: CrysAlis PRO (Rigaku OD, 2015); program(s) used to solve structure: coordinates from isotypic structure; program(s) used to refine structure: SHELXL2017/1 (Sheldrick, 2015); molecular graphics: VESTA3 (Momma \& Izumi, 2011); software used to prepare material for publication: publCIF (Westrip, 2010) and WinGX (Farrugia, 2012).

Tricalcium cobalt(II) tetraaluminate

Crystal data

$\mathrm{Ca}_{3} \mathrm{CoAl}_{4} \mathrm{O}_{10}$

$M_{r}=447.09$

Orthorhombic, $\mathrm{Pbcm}$

Hall symbol: -P 2c 2b

$a=5.1324$ (6) $\AA$

$b=16.7550(19) \AA$

$c=10.6822(12) \AA$

$V=918.60(18) \AA^{3}$

$Z=4$

Data collection

Rigaku Oxford Diffraction Xcalibur, Ruby, Gemini ultra diffractometer

Radiation source: fine-focus sealed X-ray tube, Enhance (Mo) X-ray Source

Graphite monochromator

Detector resolution: 10.3575 pixels $\mathrm{mm}^{-1}$ $\omega$ scans
$F(000)=876$

$D_{\mathrm{x}}=3.233 \mathrm{Mg} \mathrm{m}^{-3}$

Mo $K \alpha$ radiation, $\lambda=0.71073 \AA$

Cell parameters from 939 reflections

$\theta=5.0-28.4^{\circ}$

$\mu=3.97 \mathrm{~mm}^{-1}$

$T=293 \mathrm{~K}$

Fragment, colourless

$0.12 \times 0.09 \times 0.06 \mathrm{~mm}$

\section{Refinement}

Refinement on $F^{2}$

Least-squares matrix: full

$R\left[F^{2}>2 \sigma\left(F^{2}\right)\right]=0.030$

$w R\left(F^{2}\right)=0.061$

$S=1.07$

973 reflections

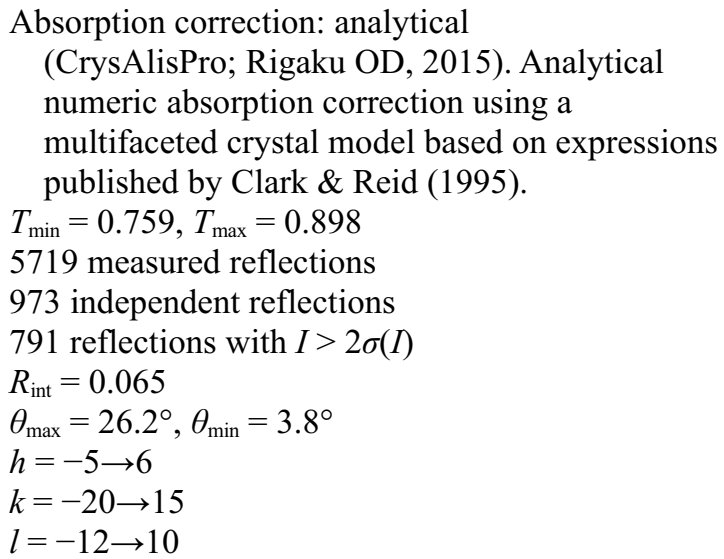

96 parameters

1 restraint$$
w=1 /\left[\sigma^{2}\left(F_{\mathrm{o}}{ }^{2}\right)+(0.0197 P)^{2}+0.4838 P\right]
$$$$
\text { where } P=\left(F_{\mathrm{o}}{ }^{2}+2 F_{\mathrm{c}}{ }^{2}\right) / 3
$$$$
(\Delta / \sigma)_{\max }<0.001
$$$$
\Delta \rho_{\max }=0.6 \mathrm{e} \AA^{-3}
$$ 
$\Delta \rho_{\min }=-0.64$ e $\AA^{-3}$
Extinction correction: SHELXL-2017/1

(Sheldrick 2015),

$\mathrm{Fc}^{*}=\mathrm{kFc}\left[1+0.001 \mathrm{xFc}^{2} \lambda^{3} / \sin (2 \theta)\right]^{-1 / 4}$

Extinction coefficient: $0.0014(4)$

Special details

Geometry. All esds (except the esd in the dihedral angle between two 1.s. planes) are estimated using the full covariance matrix. The cell esds are taken into account individually in the estimation of esds in distances, angles and torsion angles; correlations between esds in cell parameters are only used when they are defined by crystal symmetry. An approximate (isotropic) treatment of cell esds is used for estimating esds involving l.s. planes.

Fractional atomic coordinates and isotropic or equivalent isotropic displacement parameters $\left(\AA^{2}\right)$

\begin{tabular}{llllll}
\hline & $x$ & $y$ & $z$ & $U_{\text {iso }} / U_{\text {eq }}$ & Occ. $(<1)$ \\
\hline A11 & $0.63528(15)$ & $-0.07769(4)$ & $0.59968(7)$ & $0.0076(2)$ & $0.6196(19)$ \\
Co1 & $0.63528(15)$ & $-0.07769(4)$ & $0.59968(7)$ & $0.0076(2)$ & $0.3804(19)$ \\
A12 & $0.1321(2)$ & $-0.02939(7)$ & 0.75 & $0.0068(4)$ & $0.761(4)$ \\
Co2 & $0.1321(2)$ & $-0.02939(7)$ & 0.75 & $0.0068(4)$ & $0.239(4)$ \\
A13 & $0.2463(3)$ & $0.16550(9)$ & 0.75 & $0.0043(3)$ & \\
A14 & $0.3045(3)$ & 0.25 & 0.5 & $0.0062(4)$ & \\
Ca1 & $0.80287(14)$ & $0.10873(4)$ & $0.57559(7)$ & $0.0093(2)$ & \\
Ca2 & $0.7833(2)$ & $0.20768(6)$ & 0.25 & $0.0131(3)$ & \\
O1 & $0.5811(6)$ & $0.1666(2)$ & 0.75 & $0.0085(8)$ & \\
O2 & $0.0758(7)$ & $0.0742(2)$ & 0.75 & $0.0102(8)$ & \\
O3 & $0.4655(8)$ & $-0.0615(2)$ & 0.75 & $0.0192(10)$ & \\
O4 & $0.1005(5)$ & $0.21476(14)$ & $0.6201(2)$ & $0.0084(6)$ & \\
O5 & 0.5 & 0 & 0.5 & $0.0174(9)$ & \\
O6 & $0.4916(5)$ & $-0.17398(15)$ & $0.5570(2)$ & $0.0130(6)$ & \\
O7 & $-0.0172(5)$ & $-0.07854(19)$ & $0.8828(2)$ & $0.0249(7)$ & \\
& & & & & \\
\hline
\end{tabular}

Atomic displacement parameters $\left(\AA^{2}\right)$

\begin{tabular}{lllllll}
\hline & $U^{11}$ & $U^{22}$ & $U^{33}$ & $U^{12}$ & $U^{13}$ & $U^{23}$ \\
\hline A11 & $0.0068(4)$ & $0.0079(5)$ & $0.0082(4)$ & $-0.0011(3)$ & $-0.0006(3)$ & $-0.0001(3)$ \\
Co1 & $0.0068(4)$ & $0.0079(5)$ & $0.0082(4)$ & $-0.0011(3)$ & $-0.0006(3)$ & $-0.0001(3)$ \\
A12 & $0.0072(7)$ & $0.0052(7)$ & $0.0080(7)$ & $0.0001(5)$ & 0 & 0 \\
Co2 & $0.0072(7)$ & $0.0052(7)$ & $0.0080(7)$ & $0.0001(5)$ & 0 & 0 \\
A13 & $0.0042(8)$ & $0.0054(8)$ & $0.0034(7)$ & $-0.0015(6)$ & 0 & 0 \\
A14 & $0.0072(8)$ & $0.0058(8)$ & $0.0056(8)$ & 0 & 0 & $0.0003(6)$ \\
Ca1 & $0.0080(4)$ & $0.0119(4)$ & $0.0080(4)$ & $-0.0004(3)$ & $-0.0010(3)$ & $-0.0005(3)$ \\
Ca2 & $0.0116(6)$ & $0.0077(6)$ & $0.0200(6)$ & $-0.0011(5)$ & 0 & 0 \\
O1 & $0.0035(19)$ & $0.013(2)$ & $0.0085(17)$ & $0.0022(15)$ & 0 & 0 \\
O2 & $0.009(2)$ & $0.010(2)$ & $0.0120(18)$ & $0.0015(15)$ & 0 & 0 \\
O3 & $0.021(2)$ & $0.026(3)$ & $0.011(2)$ & $0.0087(19)$ & 0 & 0 \\
O4 & $0.0083(13)$ & $0.0085(15)$ & $0.0083(12)$ & $-0.0018(10)$ & $-0.0007(11)$ & $0.0021(10)$ \\
O5 & $0.017(2)$ & $0.017(2)$ & $0.018(2)$ & $-0.0078(17)$ & $0.0023(18)$ & $0.0007(17)$ \\
O6 & $0.0126(15)$ & $0.0155(16)$ & $0.0109(13)$ & $0.0068(12)$ & $-0.0013(11)$ & $-0.0033(11)$ \\
O7 & $0.0163(16)$ & $0.043(2)$ & $0.0152(14)$ & $-0.0090(14)$ & $-0.0037(13)$ & $0.0088(14)$ \\
& & & & & & \\
\hline
\end{tabular}


Geometric parameters $\left(\AA,{ }^{\circ}\right)$

\begin{tabular}{|c|c|c|c|}
\hline $\mathrm{T} 1-\mathrm{O}^{\mathrm{i}}$ & $1.794(3)$ & $\mathrm{T} 4-\mathrm{O} 4^{\mathrm{v}}$ & $1.758(2)$ \\
\hline $\mathrm{T} 1-\mathrm{O} 5$ & $1.8194(7)$ & $\mathrm{Ca} 1-\mathrm{O}^{\mathrm{iv}}$ & $2.342(3)$ \\
\hline $\mathrm{T} 1-\mathrm{O} 6$ & $1.831(3)$ & $\mathrm{Ca} 1-\mathrm{O} 1$ & $2.389(2)$ \\
\hline $\mathrm{T} 1-\mathrm{O} 3$ & $1.847(2)$ & $\mathrm{Ca} 1-\mathrm{O}^{\mathrm{vi}}$ & $2.389(3)$ \\
\hline $\mathrm{T} 2-\mathrm{O} 2$ & $1.759(4)$ & $\mathrm{Ca} 1-\mathrm{O} 4^{\mathrm{vii}}$ & $2.391(3)$ \\
\hline $\mathrm{T} 2-\mathrm{O} 3$ & $1.794(4)$ & $\mathrm{Ca} 1-\mathrm{O} 2^{\mathrm{vii}}$ & $2.402(2)$ \\
\hline $\mathrm{T} 2-\mathrm{O} 7$ & $1.810(3)$ & $\mathrm{Ca} 1-\mathrm{O} 5$ & $2.5273(8)$ \\
\hline $\mathrm{T} 2-\mathrm{O} 7^{\mathrm{ii}}$ & $1.810(3)$ & $\mathrm{Ca} 2-\mathrm{O}^{\mathrm{v}}$ & $2.348(4)$ \\
\hline T3-O1 & $1.719(4)$ & $\mathrm{Ca} 2-\mathrm{O} 4^{\text {viii }}$ & $2.503(3)$ \\
\hline T3-O2 & $1.762(4)$ & $\mathrm{Ca} 2-\mathrm{O} 4^{\mathrm{ix}}$ & $2.503(3)$ \\
\hline $\mathrm{T} 3-\mathrm{O} 4^{\mathrm{ii}}$ & $1.780(3)$ & $\mathrm{Ca} 2-\mathrm{O}^{\mathrm{vi}}$ & $2.561(3)$ \\
\hline $\mathrm{T} 3-\mathrm{O} 4$ & $1.780(3)$ & $\mathrm{Ca} 2-\mathrm{O}^{\mathrm{iv}}$ & $2.561(3)$ \\
\hline $\mathrm{T} 4-\mathrm{O} 6^{\mathrm{iii}}$ & $1.758(3)$ & $\mathrm{Ca} 2-\mathrm{O}^{3 \text { iv }}$ & $2.762(4)$ \\
\hline $\mathrm{T} 4-\mathrm{O} 6^{\mathrm{iv}}$ & $1.758(3)$ & $\mathrm{Ca} 2-\mathrm{O}^{\mathrm{iv}}$ & $2.852(3)$ \\
\hline $\mathrm{T} 4-\mathrm{O} 4$ & $1.758(2)$ & $\mathrm{Ca} 2-\mathrm{O}^{\mathrm{vi}}$ & $2.852(3)$ \\
\hline $\mathrm{O} 7^{\mathrm{i}}-\mathrm{T} 1-\mathrm{O} 5$ & $116.51(11)$ & $\mathrm{O} 2^{\mathrm{vii}}-\mathrm{Ca} 1-\mathrm{O} 5$ & $115.65(9)$ \\
\hline $\mathrm{O} 7^{\mathrm{i}}-\mathrm{T} 1-\mathrm{O} 6$ & $114.80(13)$ & $\mathrm{O} 1^{\mathrm{v}}-\mathrm{Ca} 2-\mathrm{O} 4^{\mathrm{viii}}$ & $79.73(9)$ \\
\hline $\mathrm{O} 5-\mathrm{T} 1-\mathrm{O} 6$ & $109.33(9)$ & $\mathrm{O} 1^{\mathrm{v}}-\mathrm{Ca} 2-\mathrm{O} 4^{\mathrm{ix}}$ & $79.73(9)$ \\
\hline $\mathrm{O} 7^{\mathrm{i}}-\mathrm{T} 1-\mathrm{O} 3$ & $112.30(14)$ & $\mathrm{O} 4^{\mathrm{viii}}-\mathrm{Ca} 2-\mathrm{O} 4^{\mathrm{ix}}$ & $67.34(11)$ \\
\hline $\mathrm{O} 5-\mathrm{T} 1-\mathrm{O} 3$ & $102.92(12)$ & $\mathrm{O} 1^{\mathrm{v}}-\mathrm{Ca} 2-\mathrm{O}^{\mathrm{vi}}$ & $87.38(8)$ \\
\hline $\mathrm{O} 6-\mathrm{T} 1-\mathrm{O} 3$ & $98.95(16)$ & $\mathrm{O} 4^{\mathrm{viii}}-\mathrm{Ca} 2-\mathrm{O} 6^{\mathrm{vi}}$ & $156.79(9)$ \\
\hline $\mathrm{O} 2-\mathrm{T} 2-\mathrm{O} 3$ & $116.90(19)$ & $\mathrm{O} 4^{\mathrm{ix}}-\mathrm{Ca} 2-\mathrm{O}^{\mathrm{vi}}$ & $91.52(8)$ \\
\hline $\mathrm{O} 2-\mathrm{T} 2-\mathrm{O} 7$ & $112.29(13)$ & $\mathrm{O} 1^{\mathrm{v}}-\mathrm{Ca} 2-\mathrm{O}^{\mathrm{iv}}$ & $87.38(8)$ \\
\hline $\mathrm{O} 3-\mathrm{T} 2-\mathrm{O} 7$ & $105.50(13)$ & $\mathrm{O} 4^{\text {viii }}-\mathrm{Ca} 2-\mathrm{O}^{\mathrm{iv}}$ & $91.52(8)$ \\
\hline $\mathrm{O} 2-\mathrm{T} 2-\mathrm{O}^{\mathrm{ii}}$ & $112.29(13)$ & $\mathrm{O} 4^{\mathrm{ix}}-\mathrm{Ca} 2-\mathrm{O}^{\mathrm{iv}}$ & $156.79(9)$ \\
\hline $\mathrm{O} 3-\mathrm{T} 2-\mathrm{O}^{\mathrm{ii}}$ & $105.50(13)$ & $\mathrm{O} 6^{\mathrm{vi}}-\mathrm{Ca} 2-\mathrm{O} 6^{\mathrm{iv}}$ & $107.20(12)$ \\
\hline $\mathrm{O} 7-\mathrm{T} 2-\mathrm{O}^{\mathrm{ii}}$ & $103.2(2)$ & $\mathrm{O} 1^{\mathrm{v}}-\mathrm{Ca} 2-\mathrm{O}^{\mathrm{iv}}$ & $126.24(12)$ \\
\hline $\mathrm{O} 1-\mathrm{T} 3-\mathrm{O} 2$ & $120.38(18)$ & $\mathrm{O} 4^{\text {viii }}-\mathrm{Ca} 2-\mathrm{O} 3^{\text {iv }}$ & $139.56(7)$ \\
\hline $\mathrm{O} 1-\mathrm{T} 3-\mathrm{O} 4^{\mathrm{ii}}$ & $114.55(12)$ & $\mathrm{O} 4^{\mathrm{ix}}-\mathrm{Ca} 2-\mathrm{O} 3^{\mathrm{iv}}$ & $139.56(7)$ \\
\hline $\mathrm{O} 2-\mathrm{T} 3-\mathrm{O} 4^{\mathrm{ii}}$ & $101.18(12)$ & $\mathrm{O} 6^{\mathrm{vi}}-\mathrm{Ca} 2-\mathrm{O} 3^{\mathrm{iv}}$ & $63.24(7)$ \\
\hline $\mathrm{O} 1-\mathrm{T} 3-\mathrm{O} 4$ & $114.55(12)$ & $\mathrm{O} 6^{\mathrm{iv}}-\mathrm{Ca} 2-\mathrm{O} 3^{\text {iv }}$ & $63.24(7)$ \\
\hline $\mathrm{O} 2-\mathrm{T} 3-\mathrm{O} 4$ & $101.18(12)$ & $\mathrm{O} 1^{\mathrm{v}}-\mathrm{Ca} 2-\mathrm{O}^{\mathrm{iv}}$ & $150.06(6)$ \\
\hline $\mathrm{O} 4{ }^{\mathrm{ii}}-\mathrm{T} 3-\mathrm{O} 4$ & $102.48(17)$ & 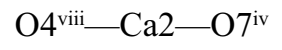 & $113.33(9)$ \\
\hline 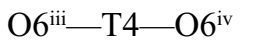 & $106.90(19)$ & $\mathrm{O} 4^{\mathrm{ix}}-\mathrm{Ca} 2-\mathrm{O} 7^{\mathrm{iv}}$ & $81.05(8)$ \\
\hline 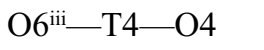 & $110.19(11)$ & $\mathrm{O} 6^{\mathrm{vi}}-\mathrm{Ca} 2-\mathrm{O} 7^{\mathrm{iv}}$ & $70.38(8)$ \\
\hline $\mathrm{O} 6^{\mathrm{iv}}-\mathrm{T} 4-\mathrm{O} 4$ & $111.36(12)$ & $\mathrm{O} 6^{\mathrm{iv}}-\mathrm{Ca} 2-\mathrm{O} 7^{\mathrm{iv}}$ & $117.70(9)$ \\
\hline 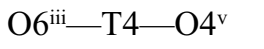 & $111.36(12)$ & $\mathrm{O}^{\mathrm{iv}}-\mathrm{Ca} 2-\mathrm{O} 7^{\mathrm{iv}}$ & $61.43(9)$ \\
\hline $\mathrm{O} 6^{\mathrm{iv}}-\mathrm{T} 4-\mathrm{O} 4^{\mathrm{v}}$ & $110.19(11)$ & $\mathrm{O} 1^{\mathrm{v}}-\mathrm{Ca} 2-\mathrm{O} 7^{\mathrm{vi}}$ & $150.06(6)$ \\
\hline $\mathrm{O} 4-\mathrm{T} 4-\mathrm{O} 4^{\mathrm{v}}$ & $106.90(18)$ & $\mathrm{O} 4^{\mathrm{viii}}-\mathrm{Ca} 2-\mathrm{O} 7^{\mathrm{vi}}$ & $81.05(8)$ \\
\hline $\mathrm{O}^{\mathrm{iv}}-\mathrm{Ca} 1-\mathrm{O} 1$ & $88.56(9)$ & $\mathrm{O} 4^{\mathrm{ix}}-\mathrm{Ca} 2-\mathrm{O} 7^{\mathrm{vi}}$ & $113.33(9)$ \\
\hline $\mathrm{O}^{\mathrm{iv}}-\mathrm{Ca} 1-\mathrm{O}^{\mathrm{vi}}$ & $82.78(10)$ & $\mathrm{O}^{\mathrm{vi}}-\mathrm{Ca} 2-\mathrm{O}^{\mathrm{vi}}$ & $117.70(9)$ \\
\hline $\mathrm{O} 1-\mathrm{Ca} 1-\mathrm{O}^{\mathrm{vi}}$ & $167.86(11)$ & $\mathrm{O}^{\mathrm{iv}}-\mathrm{Ca} 2-\mathrm{O}^{\mathrm{vi}}$ & $70.38(8)$ \\
\hline $\mathrm{O}^{\mathrm{iv}}-\mathrm{Ca} 1-\mathrm{O}^{\mathrm{vii}}$ & $100.70(9)$ & $\mathrm{O}^{\mathrm{iv}}-\mathrm{Ca} 2-\mathrm{O}^{\mathrm{vi}}$ & $61.43(9)$ \\
\hline $\mathrm{O} 1-\mathrm{Ca} 1-\mathrm{O}^{\mathrm{vii}}$ & $81.24(10)$ & $\mathrm{O} 7^{\mathrm{iv}}-\mathrm{Ca} 2-\mathrm{O} 7^{\mathrm{vi}}$ & $59.64(11)$ \\
\hline $\mathrm{O} 7^{\mathrm{vi}}-\mathrm{Ca} 1-\mathrm{O}^{\mathrm{vii}}$ & $91.99(9)$ & $\mathrm{T} 2-\mathrm{O} 2-\mathrm{T} 3$ & $140.8(2)$ \\
\hline
\end{tabular}




$\begin{array}{llll}\mathrm{O}^{\mathrm{iv}}-\mathrm{Ca} 1-\mathrm{O} 2^{\mathrm{vii}} & 163.33(10) & \mathrm{T} 2-\mathrm{O} 3-\mathrm{T} 1^{\mathrm{ii}} & 119.61(11) \\ \mathrm{O} 1-\mathrm{Ca} 1-\mathrm{O} 2^{\mathrm{vii}} & 76.74(10) & \mathrm{T} 2-\mathrm{O} 3-\mathrm{T} 1 & 119.61(11) \\ \mathrm{O} 7^{\mathrm{vi}}-\mathrm{Ca} 1-\mathrm{O} 2^{\mathrm{vii}} & 110.44(10) & \mathrm{T} 1^{\mathrm{ii}}-\mathrm{O} 3-\mathrm{T} 1 & 120.8(2) \\ \mathrm{O} 4^{\mathrm{vi}}-\mathrm{Ca} 1-\mathrm{O} 2^{\mathrm{vii}} & 69.64(11) & \mathrm{T} 4-\mathrm{O} 4-\mathrm{T} 3 & 118.32(15) \\ \mathrm{O}^{\mathrm{iv}}-\mathrm{Ca} 1-\mathrm{O} 5 & 75.31(7) & \mathrm{T} 1-\mathrm{O} 5-\mathrm{T} 1^{\mathrm{iv}} & 119.00(15) \\ \mathrm{O} 1-\mathrm{Ca} 1-\mathrm{O} 5 & 104.40(9) & \mathrm{T} 4^{\mathrm{iv}}-\mathrm{O} 6-\mathrm{T} 1 & 119.94(16) \\ \mathrm{O} 7^{\mathrm{vi}}-\mathrm{Ca} 1-\mathrm{O} 5 & 81.67(7) & \mathrm{T} 1^{\mathrm{x}}-\mathrm{O} 7-\mathrm{T} 2 & \\ \mathrm{O} 4^{\mathrm{vi}}-\mathrm{Ca} 1-\mathrm{O} 5 & 172.84(7) & & \end{array}$

Symmetry codes: (i) $x+1, y,-z+3 / 2$; (ii) $x, y,-z+3 / 2$; (iii) $-x+1, y+1 / 2, z$; (iv) $-x+1,-y,-z+1$; (v) $x,-y+1 / 2,-z+1$; (vi) $-x+1,-y, z-1 / 2$; (vii) $x+1, y, z$; (viii) $x+1,-y+1 / 2,-z+1$; (ix) $x+1,-y+1 / 2, z-1 / 2$; (x) $x-1, y,-z+3 / 2$. 